\title{
Fast radio bursts as electromagnetic radiation from cusps on superconducting cosmic strings
}

\author{
L. V. Zadorozhna* \\ Faculty of Physics, Taras Shevchenko National University of Kyiv, Glushkova ave. 4, Kyiv, 03680, Ukraine
}

\begin{abstract}
Cosmic strings are topological defects, relicts of the early Universe, which can be formed during phase transitions of fields with spontaneous broken symmetry. There also exists a special class of cosmic strings - superconducting cosmic strings, inside of which the massless charge carriers, so-called zero modes, are presented and can move along the string without any resistance. The superconducting cosmic strings during their relativistic motion through the cosmic plasma could be a powerful source of electromagnetic radiation. The emission of cusps on the superconducting strings is highly beamed and has the nature of bursts. In the present work, it is shown, that the millisecond extragalactic radio bursts, discovered during the last decade, called the fast radio bursts (FRB), with unknown nature, could be explained as radiation from cusps on superconducting cosmic strings. Estimates made for ten FRBs discovered till now show that bursts could be produced by a string with $\alpha=\Gamma G \mu / c^{2} \sim 5 \cdot 10^{-13}-10^{-12}$, where $\Gamma \sim 50$ is a dimensionless parameter, $G$ is the gravitational constant, $c$ is the speed of light, $\mu$ is the mass per unit length of a string, which corresponds to the energy scale of the phase transition $\eta \sim 1.2 \cdot 10^{12}-1.7 \cdot 10^{12} \mathrm{GeV}$. The observed duration, flux and event rate are in a good agreement with the proposed model. In the framework of emission from cusps of superconducting cosmic string loops, probability of FRB detecting is estimated, which is found to be highly close to the probability of detecting, given by observational data.
\end{abstract}

Key words: fast radio burst, superconducting cosmic strings, cosmic plasma, electromagnetic radiation

\section{INTRODUCTION}

The first extragalactic millisecond radio burst was found by a group of scientists led by Lorimer in 2007 [8] during the processing of archival data obtained by observing the Magellanic Clouds at a frequency of $1.4 \mathrm{GHz}$ using the $64-\mathrm{m}$ radio telescope Parkes in Australia. The burst was seen in the data of 24 July 2001 and it was approximately three degrees south of the Small Magellanic Cloud. The observational flux from the burst was $F_{\nu}=30 \mathrm{Jy}$ and the duration was $\tau<5 \mathrm{~ms}$. The properties of the burst did not allow to associate it with a physical phenomena that could occur in our Galaxy or in the Small Magellanic Cloud. According to current models for free electrons of cosmic plasma, the burst occurred at a distance of $r \leq 1 \mathrm{Gpc}$, corresponding to the redshift $z \leq 0.3$. The data for the subsequent 90-hour observation showed that this was a single burst phenomenon, a particular event. In 2013, it was reported that four more bursts of extragalactic nature were observed by the Parkes radio telescope. The observed phenomenon was termed "fast radio burst" (FRB). These four radio bursts FRB110220, FRB110627, FRB110703, and FRB120127 were discovered when processing data of 2011-2012, their characteristics were similar to that of Lorimer's burst

\footnotetext{
*Zadorozhna_Lida@ukr.net
}

(C) L. V.Zadorozhna, 2015 which was assigned the number FRB010724 ("spark", "Lorimer burst"). The redshifts $z$ of these radio bursts are in the range of 0.45 to 0.81 , the observed fluxes range from $F_{\nu}=0.4 \mathrm{Jy}$ to $F_{\nu}=1.3 \mathrm{Jy}$, the durations are from $1.1 \mathrm{~ms}$ up to $5.6 \pm 0.1 \mathrm{~ms}$. The width of bursts, their coordinates, duration, fluence, and distance to them are given in [14].

Recently, using the Arecibo Observatory radio telescope, another fast radio burst FRB121102 was detected in the $1.4 \mathrm{GHz}$ pulsar ALFA survey in the northern hemisphere, specifically in the region of the Galactic anti-centre. The duration of the burst was $\tau=3 \pm 0.5 \mathrm{~ms}$, the observational flux was $F_{\nu}=$ $0.4 \mathrm{Jy}$. The international team of scientists led by Laura Spitler from the Max Planck Institute of Radio Astronomy in Bonn (Germany) involved in processing FRB121102 data considered that there are seven FRBs on average every minute in the sky [13]. Since there is a set of FRBs with similar characteristics, this leads to the assumption that they are of the same nature. Several explanations for cosmological FRBs were proposed, such as a supernova explosion, bursts from magnetars, or mergers of relativistic objects black holes or neutron stars. There is also an explanation for the "Lorimer burst" FRB010724, which was proposed by Vachaspati in 2008 citevachaspati. The self-crossing and annihilation during the forma- 
tion of a cusp on a superconducting string creates the conditions for the electromagnetic radiation. The FRB was explained as the electromagnetic emission from a cusp of a superconducting string with its energy on the Grand Unification scale with the current in the string $I=10^{5} \mathrm{GeV}$.

Another work that associated cosmic strings with FRBs has explained four FRBs, which were discovered at the Parkes telescope, as electromagnetic radiation from cusps of superconducting cosmic string, that moves through a magnetic field frozen in cosmic plasma. Results obtained in this work are as follows: these radio bursts might be radiated by a string with $G \mu / c^{2} \sim 4.1 \cdot 10^{-11}$ moving through a magnetic field $B_{0}=10^{-6} \mathrm{G}[18]$. The authors also suggest to search for these strings by detection of gravitational radiation, using gravitational wave detectors such as LIGO/VIRGO or LISA. Estimates presented in [18] seem somewhat overstated both with regards to the magnitude of magnetic field and the energy scale of strings. As it will be shown below, this value of energy strings' scale could not explain such rate of detecting of FRBs in the sky, as is predicted by observations.

In general, the theory of electromagnetic radiation from the cusp of the superconducting string loop was developed by Berezinsky, Hnatyk, and Vilenkin in 2001, and such a mechanism of radiation was proposed for explaining the gamma-bursts [2].

In [5], the most comprehensive description of FRBs discovered until now is given. The authors present constraints on the origins of FRB using cosmological hydrodynamic simulations. They use these simulations to calculate contributions to the dispersion measures (DMs) of FRBs from the Milky Way, the local Universe out to $\sim 100 \mathrm{Mpc}$, the cosmological large-scale structure, and potential FRB host galaxies. They then compare the results of these simulations to the DMs for observed FRBs.

FRBs detected in real-time are of a particular interest. In [10], the authors reported the real-time discovery of a fast radio burst FRB131104 with the Parkes radio telescope in a targeted observation of the Carina dwarf spheroidal galaxy. Authors said that detailed observational analysis of the ionized gas content surrounding the Milky Way along the line of sight to FRB131104 could not reveal whether there was sufficient material to associate this FRB with CardSph at a distance of $101 \pm 5 \mathrm{kpc}$. Pending such investigations, they cannot constrain the distance to FRB131104. Hence, various possibilities for the origin of FRB131104 remain open.

Authors of [9] have reported the discovery of another radio burst in real-time. The authors have discovered FRB140514 in real-time on 14 May, 2014 at 17:14:11.06 UTC at the Parkes radio telescope, and triggered follow-up at other wavelengths within hours of the event. This FRB has celestial coordinates, which almost coincide with another radio burst FRB110220. This could indicate the single nature of this phenomenon, which is periodically repeating in time, as, for instance, bursts from cusp of string loop that arise when the loop oscillates with certain periodicity. However, some estimates for these bursts indicate that they are different, not related to one another.

In [1], authors showed that the fast radio burst FRB010621 might have a galactic nature, the upper limit on the distance was estimated as $24 \mathrm{kpc}$. However, their results also show that there is a $10 \%$ probability that FRB010621 is extragalactic.

The known data about ten discovered until now fast radio bursts (FRBs) are given in Table 1.

Cosmic strings are a type of topological defect which could have been formed during phase transitions of fields with spontaneous breaking symmetry in the early Universe. An example of such a phase transition is a phase transition in Grand Unified Theory (GUT) — separation of strong and electroweak interactions - which occurred $10^{-35} \mathrm{~s}$ after the Big Bang. Cosmic strings are quasi-one-dimensional (with diameter $d_{\mathrm{GUT}} \sim \eta_{\mathrm{GUT}}^{-1} \sim 10^{-30} \mathrm{~cm}$, where $\eta$ is the energy scale of phase transition, which in the case of the GUT is $\left.\eta=\eta_{\text {GUT }} \sim 10^{16} \mathrm{GeV}\right)$, endless (within our cosmological horizon), or closed in loops, heavy (mass per unit of length (tension) $\mu_{\mathrm{GUT}} \propto \eta_{\mathrm{GUT}}^{2} \sim 10^{22} \mathrm{~g} / \mathrm{cm}$ for GUT-strings) structures that can play an important role in various astrophysical manifestations [16]. At different stages of evolution of the Universe, the loops of cosmic strings can be responsible for the formation of structures, formation of the dark matter compressions, magnetogenesis, influencing of the ionization history of the Universe during early star formation, and can be responsible for excess of neutral hydrogen and bright spots on $21 \mathrm{~cm}$ radiation maps.

Cosmic strings can be observed through such astrophysical manifestations as gravitational microlensing of stars on closed loops, gravitational lensing on infinite strings, and radiation of electromagnetic waves and high-energy particles from kinks and cusps on the (superconducting) strings. They may also appear in the stochastic background of gravitational waves and radiation bursts of gravitational waves [7].

A large number of astrophysical manifestations are expected from superconducting cosmic strings which are the topological defects with massless carriers (zero modes) that, moving without resistance, behave like a current along the string. A significant attention is given to the electrodynamic properties of such strings and to their interaction with cosmic plasma. Except gravitational action, superconducting strings generate different types of electromagnetic radiation and particle fluxes. Especially actively these processes take place in the vicinity of cusps, periodically appearing in non-smooth (like a 
break) regions of a string, whose vertices reach the speed of light nearly instantaneously. The cusps generate sparks of electromagnetic emission directed along their motion [17] which can serve as a source of energy for some cosmological gamma bursts [2].

In articles [19, 20], authors have considered the astrophysical manifestations of superconducting cosmic strings due to the non-thermal emission of electrons of the cosmic plasma accelerated by the front of a shock wave near the magnetosphere of a string. It is shown that the superconducting strings in the magnetized intergalactic medium can be powerful sources of the non-thermal emission.

In [21] authors propose an alternative explanation for the cosmological fast radio burst FRB010724 as a synchrotron radiation of electrons accelerated at the shock front in the cusp region of superconducting cosmic strings. And, since there is approximately a uniform distribution of cosmic string loops in the Universe, further discovery of FRBs may suggest their connection to the cosmic string loops.

\section{COSMIC STRINGS IN COSMIC PLASMA}

The cosmic strings form entangled networks in the Universe that evolve in a scale-invariant way. There are two topological stable configurations of cosmic strings: infinite strings whose ends are closed somewhere under the event horizon, and closed loops. The Hubble volume contains several infinite segments of strings and a collection of loops, whose concentration changes with time as $n=1 /\left(\alpha(c t)^{3}\right)$. The average length of a loop is $l \approx \alpha c t$, where $t$ is the cosmological time moment, $\alpha$ is defined below. For analytic estimations, and since we operate with small redshifts, we consider the Friedman cosmological model with the critical density of matter (without the $\Lambda$ term), take the Hubble constant $H_{0}=72 \mathrm{~km} \cdot \mathrm{s} / \mathrm{Mpc}$, and set $t=t_{0}(1+z)^{-3 / 2}$, where $t_{0}=(2 / 3) H_{0}^{-1}$ is the Universe's lifetime, and $z$ is the redshift [3]. The parameter $\alpha$ determines the loss rate of energy by a string due to the gravitational emission, and depends on the energy scale of a phase transition, during which a string was formed:

$$
\alpha=\Gamma G \mu / c^{2}
$$

where $\Gamma \sim 50$ is a dimensionless parameter $[12,11]$. Loops oscillate periodically, different segments of the string move with different relativistic velocities, so that cusps - sharp bends-holds - are formed on the string in each period. A section of a near-cusp region at a characteristic proper (in the immovable state) distance $\Delta l$ from the cusp top are accelerated by the Lorentz factor $\gamma_{s} \sim l / \Delta l$ at the oscillatory formations of a cusp. In the reference system of the string, the near-cusp segment with the Lorentz factor $\gamma_{s}$ has the length

$$
\Delta l=l / \gamma_{s}
$$

where $\gamma_{s}=1 / \sqrt{1-\beta^{2}}$, and $\beta=v_{s} / c, v_{s}$ is the velocity of a cusp. The connection between the velocity and the size for the near-cusp region of the string depends on the specific dynamics of a loop and can be determined by numerical modelling.

Let us consider the motion of a segment of loop in the cosmic plasma with a frozen magnetic field $B_{\text {IGM }}=B_{0}(1+z)^{2}$. Oscillation of a loop in the intergalactic magnetic field is accompanied by the generation of an electric current in it with a certain mean value, averaged over the entire length [2]:

$$
I=e^{2} B_{\mathrm{IGM}} l / \hbar,
$$

where $e$ is the electron charge, $\hbar$ is the Planck constant. During the formation of a cusp, the current in the near-cusp segment increases due to the compression (a reduction of the segment length) of the string up to the value $I_{s}=I \gamma_{s}=e^{2} B_{\mathrm{IGM}} l \gamma_{s} / \hbar$.

The maximum Lorenz factor of a cusp is determined by the reverse reaction of the increasing current in the near-cusp region. The critical current value is reached, when the energy of charge carriers becomes comparable with the eigen energy of a string, i.e. with its tension $I_{\max }=e \eta / \hbar$, where $\eta \simeq\left(\mu \hbar c^{3}\right)^{1 / 2}$ is the energy scale of a phase transition, during which the loop was formed. The maximum Lorenz factor is

$$
\gamma_{s, \max }=I_{\max } / I=\frac{1}{e B_{\mathrm{IGM}} l}\left(\frac{\alpha \hbar c^{5}}{\Gamma G}\right)^{1 / 2} .
$$

Thus, the cusp region of the string will move with a very large Lorenz factor.

\section{ELECTROMAGNETIC EMISSION}

\section{FROM CUSP ON COSMIC STRING}

Electromagnetic radiation from an oscillating loop of a string can be considered as the radiation from a periodic source. The total power $P$ is expressed as a sum of the radiation power at certain harmonics [16]:

$$
P=\sum_{n} P_{n}
$$

the radiation frequency at a certain harmonic is $\nu_{n}=n / T$, where $T=l / 2 c$ is the period of oscillation. The radiation power of the $n$-th harmonic is $P_{n} \approx k I^{2} n^{-2 / 3} / c$, where $k$ is a constant coefficient that has been calculated numerically for some simple loop trajectories and is typically on the order of $k \sim 10$. The total power is [16]:

$$
P=\frac{3 k I^{2} n_{*}^{1 / 3}}{c},
$$

where $n_{*}$ is the cut-off number, which must be lower than $n_{\mathrm{em}} \sim\left(\mu / I^{2}\right)^{3 / 2}$, which is determined by comparing the maximum current in the cusp region and 
the mass per unit length (tension) of the string $\mu$. Then,

$$
n_{*}^{1 / 3}<\frac{\mu^{1 / 2} c^{2}}{3 I} .
$$

The scale of the near-cusp region is much smaller than the scale of the loop $l$, the contribution of the near-cusp region to the radiation comes mainly from higher harmonics with $n \gg 1$. The total energy of the pulse of electromagnetic radiation from the near-cusp region is $E_{\mathrm{em}}^{\text {tot }} \sim 2 k I I_{s} l / c^{2}$. An energy $E_{\mathrm{em}}^{\text {tot }} \sim n P_{n} T$ is radiated during the formation of the cusp in one oscillation period $T$ from the region $\Delta \zeta \sim n_{*}^{-1 / 3} l=l / \gamma_{s}[16]$. For a remote observer, the electromagnetic emission from a cusp region on the string is in a narrow beam with the angle between the motion direction of a cusp and the source-observer direction $\theta \sim 1 / \gamma_{s}$ and with the maximum concentration of energy in the angle $\theta_{\min } \sim 1 / \gamma_{s \text {,max }}$. The angular distribution of the radiated energy is [2]:

$$
\frac{\mathrm{d} E_{\mathrm{em}}}{\mathrm{d} \Omega} \sim \frac{k I^{2} l}{c^{2} \theta^{2}} .
$$

The isotropically-equivalent energy can be written as

$$
E_{\mathrm{em}}^{\mathrm{iso}}=E_{\mathrm{em}}^{\mathrm{tot}} \frac{4 \pi}{\Omega} \sim \frac{8 k I^{2} l}{c^{2} \theta^{3}},
$$

where $\Omega \sim \pi \theta^{2}$. The lifetime of the near-cusp region for the external observer is

$$
\tau_{c}=\frac{\alpha t_{0}}{2} \gamma_{s}^{-3}(1+z)^{-1 / 2} .
$$

The isotropically-equivalent luminosity is $L_{\mathrm{em}}^{\text {iso }}=$ $E_{\mathrm{em}}^{\text {iso }} / \tau_{c}$. The flux of the electromagnetic emission from the cusp region of the string for an external observer, if radiation propagates in a vacuum, is as follows:

$$
F_{\mathrm{em}}^{\mathrm{obs}}=\frac{L_{\mathrm{em}}^{\mathrm{iso}}(1+z)}{4 \pi d_{L}^{2}} \sim \frac{2 k I^{2} l(1+z)}{\pi d_{L}^{2} c^{2} \tau_{c} \theta^{3}}=\frac{2 P l \gamma_{s}^{2}(1+z)}{3 \pi d_{L}^{2} c \tau_{c}},
$$

where $d_{L}$ is the photometric distance from the landbased observer to the radiation region $d_{L}=3 t_{0} c(1+$ $z)^{1 / 2}\left[(1+z)^{1 / 2}-1\right]$. The dependence of the spectral flux on the frequency is

$$
F_{\nu}=F_{\nu, 0}\left(\frac{\nu}{\nu_{0}}\right)^{-2 / 3}
$$

where $F_{\nu, 0}$ is the spectral flux at the frequency $\nu_{0}$. The flux is $F_{\mathrm{em}}^{\mathrm{obs}}=\int_{\nu_{\min }}^{\nu_{\max }} F_{\nu} d \nu$, if we assume $\nu_{\min }=0$ and that the top of the cusp with $\gamma_{s \text {,max }}$ emits radiation with the frequency $\nu_{\max }$, then

$$
F_{\mathrm{em}}^{\mathrm{obs}}=\frac{1}{3} \nu_{0} F_{\nu, 0}\left(\frac{\nu_{\max }}{\nu_{0}}\right)^{1 / 3} .
$$

The spectral flux of the electromagnetic emission from the cusp region of the string for an external observer at the observational frequency $\nu_{0}$, if radiation propagates in a vacuum, is

$$
\begin{aligned}
F_{\nu, 0}=\frac{3}{\nu_{0}} F_{\mathrm{em}}^{\mathrm{obs}}\left(\frac{\nu_{\max }}{\nu_{0}}\right)^{-1 / 3}= \\
=\frac{2 P l \gamma_{s}^{2}(1+z)}{\pi d_{L}^{2} c \tau_{c} \nu_{0}}\left(\frac{\nu_{\max }}{\nu_{0}}\right)^{-1 / 3} .
\end{aligned}
$$

The observational frequency of the electromagnetic burst is inversely proportional to the intrinsic duration of the burst or the lifetime of the cusp event $\nu_{0} \equiv \nu^{\text {obs }}=1 / \tau_{c}$. The fluence, defined as the total energy per unit area of the detector, is $S=F_{\nu, 0} \tau_{c}$. Above, we suppose, that electromagnetic emission from the cusp region propagates in a vacuum, but the observed duration of radio emission $\tau^{\text {obs }} \gg \tau_{c}$ can be significantly influenced by the scattering by the turbulent IGM and the time resolution of telescopes [18]. And, as if the influence is constant, so $S=F_{\nu, 0} \tau_{c}=F^{\mathrm{obs}} \tau^{\text {obs }}$. Therefore, the spectral flux of the electromagnetic emission from the cusp region of the string for an external observer at the observational frequency $\nu^{\text {obs }}$, if radiation propagates in cosmic plasma, is

$$
F^{\mathrm{obs}}=\frac{2 P l \gamma_{s}^{2}(1+z)}{\pi d_{L}^{2} c \tau^{\mathrm{obs}} \nu^{\mathrm{obs}}}\left(\frac{\nu_{\mathrm{max}}}{\nu^{\mathrm{obs}}}\right)^{-1 / 3}
$$

and

$$
\begin{aligned}
F^{\mathrm{obs}}=\frac{2 e^{4} k B_{0}^{2} t_{0}}{3 \pi c \hbar^{2}} \frac{1}{\tau^{\mathrm{obs}} \nu^{\mathrm{obs}}} \alpha^{3} n_{*}^{1 / 3} \gamma_{s}^{2} \times \\
\times \frac{(1+z)^{-1 / 2}}{\left[(1+z)^{1 / 2}-1\right]^{2}}\left(\frac{\nu_{\mathrm{max}}}{\nu^{\mathrm{obs}}}\right)^{-1 / 3}
\end{aligned}
$$

From the formula for the duration of the cusp event, we can express

$$
\gamma_{s}=\left(\alpha t_{0}(1+z)^{-1 / 2} \nu^{\mathrm{obs}} / 2\right)^{1 / 3}
$$

Let us suppose that a cosmic string with $\alpha=$ $10^{-12}$ moves in cosmic plasma with a magnetic field $B_{0}=10^{-10} \mathrm{G}$ and emits radiation that we can detect at the frequency $\nu^{\text {obs }}=1.3 \mathrm{GHz}$. The maximum Lorenz factor of the cusp from equation (1) is $\gamma_{s, \max }=7 \cdot 10^{8}(1+z)^{-1 / 2}$ and the Lorenz factor from equation (3) is much smaller, $\gamma_{s}=6.5 \cdot 10^{4}(1+z)^{-1 / 6}$. It should be noted that the maximum Lorentz factor is achieved only once, at the first time during the formation of the cusp. The following times of cusp 
formation, as string loses its energy during oscillations, the near-cusp region is smoothed and has the much smaller Lorentz factor. We assume that the first appearance of the cusp on the string was a long time ago, and the Lorentz factor $\gamma_{s}$ is close to the maximum Lorentz factor, to which the top of the cusp can be accelerated now. For the same reason, the near-cusp region does not emit radiation with the frequency $\nu_{\max }$ and we assume that the maximum frequency is close to $\nu^{\text {obs }}$. And, since $n_{*}^{-1 / 3} l=l / \gamma_{s}$, from equation (2), we have

$$
F^{\mathrm{obs}}=\frac{e^{4} k B_{0}^{2} t_{0}}{3 \pi c \hbar^{2}} \frac{1}{\tau^{\mathrm{obs}}} \alpha^{4} \frac{(1+z)^{-1}}{\left[(1+z)^{1 / 2}-1\right]^{2}},
$$

the spectral flux for an external observer does not depend on the observational frequency. The dependence between observed fluxes from cusps $F^{\mathrm{obs}}$ and redshifts $z$ where bursts are observed is shown for different parameters: $\alpha=10^{-12}, \tau^{\text {obs }}=1 \mathrm{~ms} ; \alpha=$ $10^{-12}, \tau^{\text {obs }}=5 \mathrm{~ms} ;$ and $\alpha=5 \cdot 10^{-13}, \tau^{\text {obs }}=5 \mathrm{~ms}$ for magnetic field magnitude $B_{0}=10^{-10} \mathrm{G}$ in Fig.1. As if there is a certain probability that FRB010621, FRB131104 are extragalactic FRB, we estimated the fast radio bursts's redshifts, where these bursts possibly have taken place (see Fig. 1).

\section{FRBS AS EMISSION FROM CUSP ON SUPERCONDUCTING COSMIC STRING}

Using the observational cosmological data for FRBs, we can find the characteristics of superconducting cosmic strings. For example, the FRB110220 has the following characteristics: the detection frequency is $\nu^{\text {obs }}=1.3 \mathrm{GHz}$, the redshift is $z=0.81$, the observational duration is $\tau^{\text {obs }}=5.6 \cdot 10^{-3} \mathrm{~ms}$ and the peak flux is $\left.F^{\mathrm{obs}}=1.3 \cdot 10^{-23} \mathrm{erg} \mathrm{cm}^{-2} \mathrm{c}^{-1} \mathrm{~Hz}^{-1}\right)$. Assuming the magnetic field is equal to $B_{0}=$ $10^{-10} \mathrm{G}$, we obtain from the equations $(2),(4)$ :

$$
F^{\mathrm{obs}}=0.013 \alpha^{3} \gamma_{s}^{3}=2.7 \cdot 10^{24} \alpha^{4} \mathrm{erg} \mathrm{cm}^{-2} \mathrm{c}^{-1} \mathrm{~Hz}^{-1} \text {, }
$$

for the FRB110220, $\alpha=1.5 \cdot 10^{-12}$, the Lorenz factor of the cusp region is $\gamma_{s}=6.2 \cdot 10^{4}$. Similarly, the value $\alpha \sim 5 \cdot 10^{-13}-10^{-12}$ is obtained for other FRBs (see Table 1).

The characteristics of the superconducting cosmic string loop $\alpha, \gamma_{s}$ if it emits the electromagnetic burst moving in intergalactic medium with the magnetic field $B_{0}=10^{-8} \mathrm{G}$ are presented in Table 1 .

If we suppose that all loops of superconducting cosmic strings are formed during the same phase transition of fields with spontaneous breaking symmetry in the early Universe, and they have $\alpha \sim$ $10^{-12}$, but on the other hand, different loops move in cosmic plasma with different strength of frozen magnetic field $B_{0}$ and may have different size $l=p \alpha c t$, where $p$ is the normalization coefficient (above we considered that all of the loops have the same average size $l=\alpha c t$ ), we can find the multiplication of two parameters $p^{2} B_{0}$, from observational data for FRBs, using the relation

$$
F^{\mathrm{obs}}=3.2 \cdot 10^{-7} \frac{1}{\tau^{\mathrm{obs}}} p^{4} B_{0}^{2} \frac{(1+z)^{-1}}{\left[(1+z)^{1 / 2}-1\right]^{2}} .
$$

The results are presented in Table 1.

\section{DETECTION PROBABILITIES OF FRBS}

The lead author of the paper [13], Laura Spitler from Max Planck Institute for Radio Astronomy (MPIfR) in Bonn, who was involved in the processing of data on FRBs, considered that on average every minute in the sky there are seven FRBs $N \approx 7 \mathrm{~min}^{-1}=420 \mathrm{hr}^{-1}$. We will further use this assumption.

The rate of FRBs originating at cusps in the redshift interval $\mathrm{d} z$ and seen at the angle $\theta$ in the interval $\mathrm{d} \theta$ is given by [2]:

$$
\mathrm{d} N \sim f_{B} \cdot \frac{1}{2} \theta \mathrm{d} \theta(1+z)^{-1} \nu(z) d V(z) .
$$

We assume that the fraction of volume of the Universe occupied by magnetized plasma is $f_{B} \sim 0.1$, the number of cusp events in unit spacetime volume is $\nu(t) \sim n_{l}(t) / T \sim 2 \alpha^{-2} c^{-3} t^{-4}$, the oscillation period of a loop is $T \sim \alpha t / 2$, the proper volume between redshifts $z$ and $\mathrm{d} z$ is $\mathrm{d} V=54 \pi(c t)^{3}(1+$ $z)^{-11 / 2}\left[(1+z)^{1 / 2}-1\right]^{2} \mathrm{~d} z$, and we use the relations $t=t_{0}(1+z)^{-3 / 2}$. Then,

$\mathrm{d} N \sim 54 \pi f_{B} \cdot \frac{1}{\alpha^{2} t_{0}} \theta \mathrm{d} \theta(1+z)^{-1 / 2}\left[(1+z)^{1 / 2}-1\right]^{2} \mathrm{~d} z$.

It is important to comply with a certain order of integration, namely, first we integrate over $\theta$ between 0 and $1 / \gamma_{s}$, as $\gamma_{s}$ depends on $z$, then over $z$ from 0 to 2 (taken little overestimated; we consider that all bursts that can be registered have $z \leq 2$ ). This yields

$$
\begin{aligned}
\mathrm{d} N \sim 27 \pi f_{B} & \left(\frac{2}{\nu^{\mathrm{obs}} t_{0}^{5 / 2}}\right)^{2 / 3} \frac{1}{\alpha^{8 / 3}} \times \\
& \times \int_{0}^{2}(1+z)^{-1 / 6}\left[(1+z)^{1 / 2}-1\right]^{2} \mathrm{~d} z .
\end{aligned}
$$

For $\nu^{\text {obs }}=1.3 \mathrm{GHz}$, we find $N=9.68 \cdot 10^{-34} \times$ $\alpha^{-8 / 3} \min ^{-1}$. So, in order to explain such number of FRBs, the string must have $\alpha=2 \cdot 10^{-13}$. The linear density of this string is $\mu=\alpha c^{2} / \Gamma G=5.2 \cdot 10^{13} \mathrm{~g} / \mathrm{cm}$, 
which corresponds to the energy scale of the phase transition $\eta=1.2 \cdot 10^{9} \mathrm{erg}=7.6 \cdot 10^{11} \mathrm{GeV}$, the oscillation period of a loop or a repetition rate of the bursts is $T \sim \alpha t / 2=\alpha t_{0}(1+z)^{-3 / 2} / 2=0.5(1+z)^{-3 / 2}$ days.

The rates of FRBs from $z=0 . .2$ estimated in this way are presented in Table 2 for different values of the parameter $\alpha$.

\section{CONCLUSIONS}

In the past decade, a new class of objects, which received the name of the FRBs, was discovered at the Parkes telescope and the Arecibo observatory. There are 10 observed FRBs at the moment. All of them have similar characteristics: the frequency of observations is $1.3 \mathrm{GHz}$ and $1.4 \mathrm{GHz}$, the duration is approximately several milliseconds, and the peak of observed flux is approximately several Jy.

Various theories exist attempting to explain this phenomenon. We propose to consider the FRBs as electromagnetic emission from the cusps of superconducting cosmic strings, which move with a large Lorentz factor through the magnetic field, frozen into cosmic plasma. We show in this work that it is possible to register radiation from cusps of cosmic string loops at Earth's orbit. The radiation flux is periodic, highly beamed, and has the nature of a burst. Significant collimation decreases the possibility of observing a single phenomenon from the Earth, even so collimation effects are compensated for a number of parameters by a large number of loops in a region accessible for observations.

In the present work, the characteristics of 10 FRBs discovered until now are studied. Also, the main characteristics of cosmic strings, which can serve as a source of these bursts, are explored. Under the assumption of an average loop's length, we found the energy characteristic of a string $\alpha=\Gamma G \mu / c^{2}$, which is related to the energy of phase transition, during which these strings were created. In tabular form, the values of the parameter $\alpha$ are given for two magnitudes of the magnetic field, where superconducting string loops move: $B_{0}=10^{-10} \mathrm{G}$ and $B_{0}=$ $10^{-8} \mathrm{G}$. For the variant that seems to be the most realistic, namely, a magnetic field $B_{0}=10^{-10} \mathrm{G}$, we have obtained the value $\alpha=\Gamma G \mu / c^{2} \sim 5 \cdot 10^{-13}$ $10^{-12}$, which corresponds to the energy scale of the phase transition $\eta \sim 1.2 \cdot 10^{12}-1.7 \cdot 10^{12} \mathrm{GeV}$. The dependencies between the flux from cusps and redshifts, where bursts are observed, are shown for several sets of parameters: $\alpha=10^{-12}, \tau^{\text {obs }}=1 \mathrm{~ms} ; \alpha=$ $10^{-12}, \tau^{\text {obs }}=5 \mathrm{~ms} ;$ and $\alpha=5 \cdot 10^{-13}, \tau^{\text {obs }}=5 \mathrm{~ms}$ for magnetic field magnitude $B_{0}=10^{-10} \mathrm{G}$ (Fig. 1). There is a certain probability that the fast radio bursts FRB010621, FRB131104 are extragalactic. In framework of our model, we have estimated the redshifts, where these bursts have possibly taken place.

In the framework of the theory of emission from cusps of the superconducting cosmic string loops, we have also estimated the probability of FRB detection, that is highly close to the probability of detection, given by the observational data. (Namely, $N \approx 7 \mathrm{~min}^{-1}=3679200$ year $^{-1}$ ).

Radiation from cusps of the superconducting cosmic strings could also be explained by the nonthermal emission of electrons of cosmic plasma accelerated on the shock wave front around the nearcusp region of a string. Such a shock wave will be generated during the relativistic motion of superconducting cosmic string's segment through the cosmic plasma with a magnetic field frozen in it.

\section{ACKNOWLEDGEMENTS}

The author thanks the anonymous reviewer for his or her constructive comments, which helped to improve the present paper.

\section{REFERENCES}

[1] Bannister K. W. \& Madsen G. J. 2014, MNRAS, 440, 353

[2] Berezinsky V., Hnatyk B. \& Vilenkin A. 2001, Phys. Rev. D, 64, 043004

[3] Berezinsky V., Olum K. D., Sabancilar E. \& Vilenkin A., 2009, Phys. Rev. D, 80, 023014

[4] Burke-Spolaor S. \& Bannister K. W. 2014, ApJ, 792, 19

[5] Dolag K., Gaensler B. M., Beck A. M. \& Beck M. C. 2014, MNRAS, 451, 4277

[6] Keane E. F., Stappers B. W., Kramer M. \& LyneA. G. 2012, MNRAS, 425, L71

[7] Long A. J. \& Vachaspati T. 2014, JCAP, 12, 040

[8] Lorimer D. R., Bailes M., McLaughlin M. A., Narkevic D. J. \& Crawford F. 2007, Science Express, 318, 777

[9] PetroffE., Bailes M., Barr E.D. et al. 2014, MNRAS, 447, 246

[10] Ravi V., Shannon R. M. \& Jameson A. 2014, ApJ, 799, L5

[11] Ringeval C., Sakellariadou M. \& Bouchet F. R. 2007, JCAP, 02, 023

[12] Siemens X., Creighton J., Maor I. et al. 2006, Phys. Rev. D, 73, 105001

[13] Spitler L. G., Cordes J. M., Hessels J. W. T. et al. 2014, ApJ, 790, 101

[14] Thornton D., Stappers B., Bailes M. et al. 2013, Science, 341,53

[15] Vachaspati T. 2008, Phys. Rev. Lett. 101, 141301

[16] Vilenkin A. \& Shellard E.P.S. 1994, 'Cosmic Strings and Other Topological Defects', Cambridge Univ. Press, Cambridge

[17] Vilenkin A. \& Vachaspati T. 1987, Phys. Rev. Lett., 58, 1041

[18] Yu Y.-W., Cheng K.-S., Shiu G. \& Tye H. 2014, JCAP, 11,040

[19] Zadorozhna L. V. \& Hnatyk B. I. 2009, Ukrainian Journal of Physics, 54, 1044

[20] Zadorozhna L. V. \& Hnatyk B. I. 2009, Ukrainian Journal of Physics, 54, 1152

[21] Zadorozhna L.V. \& Hnatyk B.I., 2015, Visnyk Kyivskogo Universytetu. Astronomia, 61, 15 
Table 1: Observed properties of ten published FRBs. Here, $\ell, b$ are Galactic coordinates centred on $\ell=0^{\circ}, b=0^{\circ}, F_{\nu}$ is the observational peak flux, $\nu^{\text {obs }}$ is the frequency, $z$ is the redshift, $\tau^{\text {obs }}$ is the time width, references are given in column ref, the multiplication of the two parameters $p^{2} B_{0}$ for strings with $\alpha=10^{-12}$, i. e. the normalization coefficient in the length of loop $p$ and the magnetic field strength in intergalactic medium $B_{0}$. The parameter $\alpha$, the Lorenz factor $\gamma_{s}^{\prime}$ are calculated for the magnetic field $B_{0}=10^{-8} \mathrm{G}$ and the parameter $\alpha$, the Lorenz factor $\gamma_{s}$ is for $B_{0}=10^{-10} \mathrm{G}$.

\begin{tabular}{|c|c|c|c|c|c|c|c|c|c|c|c|c|}
\hline FRB & $\begin{array}{c} \\
\left({ }^{\circ}\right)\end{array}$ & $\begin{array}{c}b \\
\left({ }^{\circ}\right)\end{array}$ & $\begin{array}{c}F_{\nu} \\
(\mathrm{Jy})\end{array}$ & $\begin{array}{c}\nu^{\mathrm{obs}} \\
(\mathrm{GHz})\end{array}$ & $z$ & $\begin{array}{l}\tau^{\mathrm{obs}} \\
(\mathrm{ms})\end{array}$ & ref & $\begin{array}{c}p^{2} B_{0} \\
\times 10^{-10}(\mathrm{G})\end{array}$ & $\begin{array}{c}\alpha \\
\times 10^{-13}\end{array}$ & $\begin{array}{c}\dot{\gamma}_{s} \\
\times 10^{4}\end{array}$ & $\begin{array}{c}\alpha \\
\times 10^{-12}\end{array}$ & $\begin{array}{c}\gamma_{s} \\
\times 10^{4}\end{array}$ \\
\hline 010621 & 25.4 & -4.0 & 0.4 & 1.4 & $?$ & 7.8 & [6] & - & - & - & - & - \\
\hline 010724 & 300.8 & -41.9 & 30 & 1.4 & 0.12 & 4.6 & [8] & 1.3 & 1.2 & 3.0 & 1.2 & 6.4 \\
\hline 011025 & 356.6 & -20.0 & 0.3 & 1.4 & 0.69 & $<9.4$ & [4] & 1.2 & 1.1 & 2.9 & 1.1 & 6.3 \\
\hline 110220 & 50.8 & -54.7 & 1.3 & 1.3 & 0.81 & $5.6 \pm 0.1$ & [14] & 2.2 & 1.5 & 2.8 & 1.5 & 6.2 \\
\hline 110627 & 355.8 & -41.7 & 0.4 & 1.3 & 0.61 & $<1.4$ & {$[14]$} & 0.5 & 0.66 & 2.4 & 0.66 & 5.3 \\
\hline 110703 & 81.0 & -59.0 & 0.5 & 1.3 & 0.96 & $<4.3$ & [14] & 1.4 & 1.2 & 2.9 & 1.2 & 6.2 \\
\hline 120127 & 49.2 & -66.2 & 0.5 & 1.3 & 0.45 & $<1.1$ & [14] & 0.3 & 0.57 & 2.3 & 0.57 & 5.1 \\
\hline 121102 & 175.0 & -0.2 & 0.4 & 1.4 & 0.26 & $3 \pm 0.5$ & [13] & 0.3 & 0.51 & 2.4 & 0.51 & 5.2 \\
\hline 131104 & 260.6 & -21.9 & 1.1 & 1.4 & $?$ & $<0.64$ & {$[10]$} & - & - & - & - & - \\
\hline 140514 & 50.8 & -54.6 & 0.47 & 1.4 & 0.44 & 2.8 & {$[9]$} & 0.5 & 0.7 & 2.6 & 0.7 & 5.6 \\
\hline
\end{tabular}

Table 2: The rate of FRBs $N$ in the redshift interval $z=0 \ldots 2$ which might be detected at $\nu^{\text {obs }}=1.3 \mathrm{GHz}$ from cusps for different values of the parameter $\alpha$. The linear density of string is $\mu$, the energy scale of phase transition is $\eta$, the oscillation period of the loop is $T$.

\begin{tabular}{ccccc}
\hline$\alpha$ & $\mu$ & $\eta$ & $T$ & $N(z=0 \ldots 2)$ \\
& $(\mathrm{g} / \mathrm{cm})$ & $(\mathrm{GeV})$ & $\times(1+z)^{-3 / 2}($ days $)$ & events $/$ year \\
\hline $2 \cdot 10^{-13}$ & $5.2 \cdot 10^{13}$ & $7.6 \cdot 10^{11}$ & 0.504 & 3679200 \\
$5 \cdot 10^{-13}$ & $1.4 \cdot 10^{14}$ & $1.2 \cdot 10^{12}$ & 1.26 & 323149 \\
$10^{-12}$ & $2.7 \cdot 10^{14}$ & $1.7 \cdot 10^{12}$ & 2.52 & 50892 \\
$5 \cdot 10^{-12}$ & $1.4 \cdot 10^{15}$ & $3.9 \cdot 10^{12}$ & 12.6 & 698.1 \\
$10^{-11}$ & $2.7 \cdot 10^{15}$ & $5.5 \cdot 10^{12}$ & 25.2 & 109.7 \\
$5 \cdot 10^{-11}$ & $1.4 \cdot 10^{16}$ & $7.7 \cdot 10^{12}$ & 125.9 & 1.50 \\
$10^{-10}$ & $2.7 \cdot 10^{16}$ & $1.7 \cdot 10^{13}$ & 251.9 & 0.24 \\
$5 \cdot 10^{-10}$ & $1.4 \cdot 10^{17}$ & $3.9 \cdot 10^{13}$ & 1259.3 & $3.2 \cdot 10^{-3}$ \\
\hline
\end{tabular}




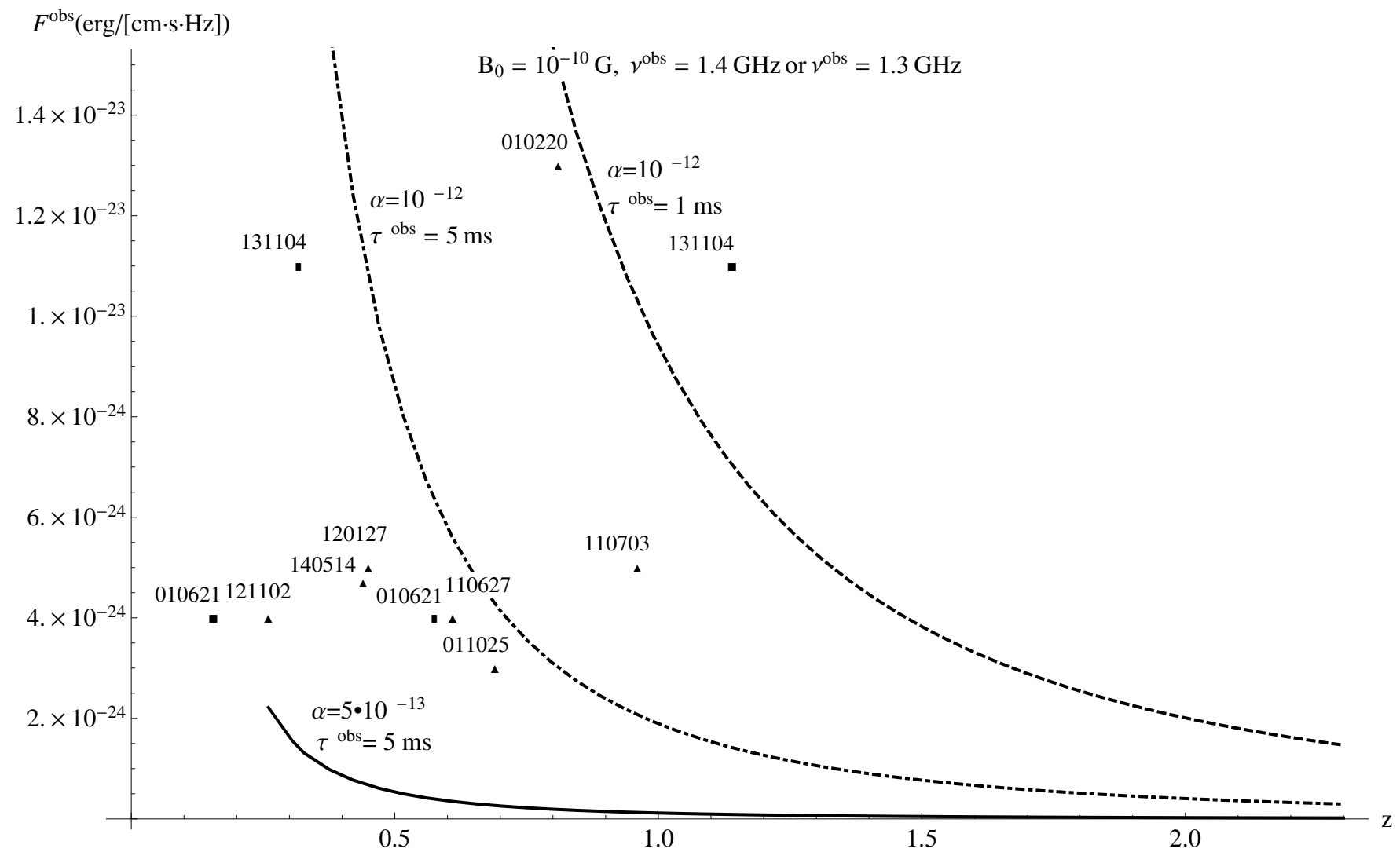

Fig. 1: The dependence between observed electromagnetic flux from cusps $F^{\text {obs }}$ and redshifts where bursts are observed $z$ for intergalactic magnetic field $B_{0}=10^{-10} \mathrm{G}$ for $\alpha=10^{-12}, \tau^{\text {obs }}=1 \mathrm{~ms}$ (dashed line), for $\alpha=10^{-12}, \tau^{\text {obs }}=5 \mathrm{~ms}$ (dot-dashed line) and for $\alpha=5 \cdot 10^{-13}, \tau^{\text {obs }}=5 \mathrm{~ms}$ (solid line). Fast radio bursts FRB121102, FRB140514, FRB120127, FRB110627, FRB011025, FRB110220, FRB110703 are marked as triangles. FRB010724 is a bit out of the general picture because of high flux $F_{\nu}=30 \mathrm{Jy}$. For the fast radio bursts FRB010621, FRB131104 we estimated the redshifts where these bursts possibly arose. They are marked as squares if they were emitted by string with $\alpha=10^{-12}$ and marked as rectangles if they were emitted by string with $\alpha=5 \cdot 10^{-13}$. 